\title{
Comparação dos aspectos da autoimagem e domínios da qualidade de vida em idosos praticantes e não praticantes de pilates em Curitiba, Paraná
}

\author{
Comparison of self-image aspects and quality of life domains in elderly practitioners and \\ non-practitioners of pilates in Curitiba, Paraná \\ Comparación de aspectos de autoimagen y dominios de calidad de vida entre ancianos \\ practicantes y no practicantes de pilates en Curitiba (Brasil)
}

Cinthia Fernanda da Fonseca Silva', Crismarie Casper Hackenberg², Taís Glauce Fernandes de Lima Pastre ${ }^{3}$, Valdomiro de Oliveira ${ }^{4}$, Gislaine Cristina Vagetti ${ }^{5}$

RESUMOI O objetivo deste estudo foi comparar os aspectos da autoimagem e domínios da qualidade de vida em idosos praticantes e não praticantes de pilates na cidade de Curitiba, Paraná, Brasil. O estudo foi quantitativo, descritivo, comparativo e com delineamento transversal. A população da pesquisa foi formada por 93 idosos, após cálculo amostral com tamanho de efeito $f(0,43)$. A amostra foi intencional e os participantes foram divididos em três grupos: grupo pilates, grupo hidroginástica e grupo não praticante. A pesquisa foi realizada por aplicação de questionário sociodemográfico, econômico, autoimagem, WHOQOL-BREF e OLD. A análise estatística foi feita por meio de avaliação comparativa com Anova, teste qui-quadrado e post hoc de Bonferroni. A comparação da autoimagem entre os grupos não mostrou nenhuma diferença significativa $\left(X^{2}=3,72, p=0,15\right)$, apesar de o número de participantes com baixa autoimagem ter sido alto. Nos domínios da qualidade de vidal, os grupos pilates e hidroginástica apresentaram maiores valores de média, em geral. No WHOQOL-OLD, foram identificadas diferenças significativas no domínio autonomia $\left(F_{(2,90)}=\right.$ $7,46, p=0,001$ ) entre os grupos pilates e hidroginástica, quando comparados ao grupo não praticantes. No domínio morte e morrer $\left(F_{(2,90)}=4,46, p=0,01\right)$, os maiores valores foram no grupo não praticantes em relação ao grupo pilates. Os resultados sugerem que a prática de atividade física pode beneficiar os idosos na questão da qualidade de vida, porém não isoladamente.

Descritores|Envelhecimento; Autoimagem; Qualidade de Vida; Idoso; Técnicas de Exercício e de Movimento.

ABSTRACT| This quantitative and descriptive cross-sectional study compares the self-image aspects and quality of life domains in older adults practitioners and non-practitioners of pilates in the city of Curitiba, Paraná. After sample calculation with effect size $\mathrm{f}$ (0.43), an intentional sampling was conducted, resulting in a study population of 93 older adults divided into three groups: pilates group, water aerobics group, and non-practicing group. Data was collected using a sociodemographic, economic, and self-image questionnaire, and the instruments WHOQOL-BREF and OLD. Statistical comparative analysis was performed using Anova, Chisquare test, and Bonferroni post hoc. Although the number of participants with low self-image was high, the comparison of this variable between groups showed no significant difference $\left(X^{2}=3.72, p=0.15\right)$. Regarding the quality of life domains, the pilates and water aerobics groups had higher average values, in general. The WHOQOL-OLD instruments identified significant differences in the Autonomy domain $\left(F_{(2.90)}=7.46\right.$, $p=0.001$ ) between the pilates and water aerobics groups when compared to the non-practicing group. As for the Death and Dying domain $\left(F_{(2.90)}=4.46, p=0.01\right)$, the non-practicing group

Universidade Federal do Paraná (UFPR) - Curitiba (PR), Brasil. E-mail: fisioterapiacinthia@gmail.com. ORCID-0000-0002-6519-3857 2Universidade Federal do Paraná (UFPR) - Curitiba (PR), Brasil. E-mail: crismarie@rioacappella.com.br. ORCID-0000-0003-3838-0769 3Universidade Federal do Paraná (UFPR) - Curitiba (PR), Brasil. E-mail: tpastre7@gmail.com. ORCID-0000-0002-7832-6372 ${ }^{4}$ Universidade Federal do Paraná (UFPR) - Curitiba (PR), Brasil. E-mail: oliveirav457@gmail.com. ORCID-0000-0002-8709-8471 5Universidade Estadual do Paraná (Unespar) - Curitiba (PR), Brasil. E-mail: gislainevagetti@hotmail.com. ORCID-0000-0003-0704-1297 
showed the highest values in relation to the pilates group. Results suggest that physical exercise can benefit older adults regarding quality of life, but not in isolation.

Keywords|Aging. Self Concept; Quality of Life; Exercise Movement Techniques.

RESUMEN| El objetivo de este estudio fue comparar los aspectos de autoimagen y los dominios de calidad de vida entre ancianos practicantes y no practicantes de pilates en la ciudad de Curitiba, Paraná, Brasil. Este es un estudio cuantitativo, descriptivo, comparativo y con diseño transversal. La población de investigación estuvo constituida por 93 ancianos después del cálculo de la muestra con tamaño del efecto $f(0,43)$. La muestra fue intencional, y se dividieron a los participantes en tres grupos: grupo de pilates, grupo de hidrogimnasia y grupo de no practicantes. Se les aplicó un cuestionario sociodemográfico, económico, de autoimagen, WHOQOL-BREF y WHOQOL-OLD. El análisis estadístico se realizó mediante evaluación comparativa utilizando Anova, prueba de chi-cuadrado y prueba de post hoc de Bonferroni. La comparación de la autoimagen entre los grupos no mostró ninguna diferencia significativa $\left(X^{2}=3,72, p=0,15\right)$, aunque el número de participantes con baja autoimagen fue alto. En los dominios de la calidad de vida, los grupos de pilates y de hidrogimnasia presentaron, por lo general, una media de valores más alta. En WHOQOL-OLD, hubo diferencias significativas en el dominio de autonomía $\left(F_{(2,90)}=7,46, p=0,001\right)$ entre los grupos de pilates y de hidrogimnasia en comparación con el grupo de no practicantes. En el dominio muerte y morir $\left(F_{(2,90)}=4,46, p=0,01\right)$, los valores más altos se encontraron en el grupo de no practicantes en comparación con el grupo de pilates. Los resultados evidencian que la práctica de actividad física puede beneficiar a las personas mayores en términos de calidad de vida, pero no de forma aislada.

Palabras clave| Envejecimiento; Autoimagen; Calidad de Vida; Anciano; Técnicas de Ejercicio con Movimientos.

\section{INTRODUÇÃO}

A longevidade pode ser considerada uma história de sucesso para a humanidade. Alcançar a velhice, antes privilégio dos países desenvolvidos, hoje pode acontecer mesmo nos países em desenvolvimento. Os idosos representam atualmente $12 \%$ da população mundial, com previsão de duplicar até 2050 e triplicar em $2100^{1}$. Segundo o $\mathrm{IBGE}^{2}, 25,5 \%$ da população brasileira deverá ter mais de 65 anos até 2060. Assim, a maior conquista do século $\mathrm{XX}$ se transformou em um grande desafio, pois o crescente envelhecimento da população exige a incorporação de qualidade e programas de saúde no prolongamento da vida ${ }^{3}$.

O envelhecimento é um processo fisiológico, biológico e psicológico que afeta a capacidade funcional do indivíduo, iniciando-se no nascimento e continuando até a morte. Nas alterações inerentes ao processo de envelhecimento, se encaixa a autoimagem ${ }^{4,5}$. A autoimagem é o reconhecimento que cada indivíduo faz de si mesmo, como sentem suas potencialidades, sentimentos, atitudes e ideias ${ }^{6}$. Ela consiste em quatro componentes que estão envolvidos em toda a ação: movimento, sensação, sentimento e pensamento. Por isso, cada pessoa fala, se move, pensa e sente de distintas maneiras ${ }^{7}$.

A imagem corporal começa a se formar desde o nascimento, sendo o controle motor dos membros a primeira impressão que se tem do corpo ${ }^{8}$. O corpo é movido por reações fisiológicas e quando alguma delas está prejudicada, a autoimagem pode ser afetada, interferindo na qualidade de vida do indivíduo ${ }^{4}$.

A qualidade de vida transita por diferentes áreas de conhecimento e possui múltiplas dimensões, como a física, a psicológica e a social, sendo que cada uma comporta vários aspectos ${ }^{9}$. Para conceituação da qualidade de vida, é necessário observar inúmeros elementos do cotidiano do ser humano, desde a percepção e expectativa subjetivas sobre a vida até questões mais deterministas, como o agir clínico frente a doenças, enfermidades e a questão entre mente e corpo $^{10,11}$.

Joseph Pilates criou seu método, a Contrologia, no início do século XX. Para ele, corpo e mente deveriam funcionar juntos e não colocados um contra o outro, o que resultaria na concentração de apenas uma das partes, limitando a outra. Ele buscava a completa coordenação entre corpo e mente ${ }^{12}$.

A prática de exercícios físicos sem atenção plena leva ao declínio da consciência corporal na vida adulta e idosa, sendo que Joseph Pilates pensava que somente por meio de uma boa educação corporal é que seria possível corrigir os maus hábitos. Por isso, é importante que a educação em consciência corporal seja trabalhada desde cedo, para que ocorra o desenvolvimento uniforme do corpo, vitalidade e maior qualidade de vida no envelhecimento ${ }^{13}$.

Uma revisão integrativa que buscou produções científicas publicadas sobre imagem corporal e autoestima em idosos encontrou 14 artigos. Entre eles, apenas três utilizaram o questionário de autoimagem e autoestima de Steglich, 
voltado para a população idosa. Essa informação pode contribuir na compreensão do motivo de haver poucos estudos sobre o tema autoimagem e idosos na literatura ${ }^{14}$.

O praticante do método pilates aprende a sentir seus movimentos, a ter autopercepção corporal ao executálos e explorar variações ao mover-se. Ao comparar com praticantes de outras modalidades de exercícios, identificase que a prática do pilates beneficia os seus praticantes, pois proporciona maior consciência corporal e melhora na atenção plena. Isso evidencia a hipótese de que a autoimagem e a qualidade de vida dos idosos praticantes de pilates seria melhor em comparação aos adeptos de outras modalidades de atividade física e não praticantes ${ }^{15,16}$. Assim, o objetivo desta pesquisa foi comparar a influência do método pilates na autoimagem e qualidade de vida de idosos que praticam e não praticam a atividade.

\section{METODOLOGIA}

Esse é um estudo com enfoque quantitativo, descritivo, comparativo e transversa ${ }^{17,18}$. A população para este estudo foi caracterizada por 93 idosos, com idade superior ou igual a 60 anos. A amostra foi intencional e os participantes foram divididos em três grupos: grupo pilates, grupo hidroginástica e grupo não praticante (idosos que se reuniam em encontros de convivência). Os locais selecionados para a coleta de dados foram três estúdios de pilates, uma academia de ginástica e uma comunidade religiosa. A comunidade religiosa foi escolhida por ter um grupo de convivência social de idosos que se reunia toda a semana, sem o intuito de praticar atividade física. Todos os ambientes localizados em Curitiba-PR.

Os critérios de inclusão foram participantes com idade igual ou superior a 60 anos, do sexo feminino e masculino, alfabetizados e que aceitaram participar da pesquisa. Como critérios de exclusão, pessoas com idade inferior a 60 anos e que não entregaram assinado o termo de consentimento livre e esclarecido. $O$ projeto e os procedimentos de coleta de dados seguiram todas as exigências da Resolução no 466/2012 do Conselho Nacional de Saúde (CNS), sendo executados após aprovação pelo Comitê de Ética, recebendo a autorização com número do parecer 3.073.628 e CAAE 02549018.5.0000.0094.

\section{Coleta de dados}

A pesquisa foi realizada por meio de perguntas fechadas aos participantes, utilizando os seguintes questionários: questionário sociodemográfico ${ }^{19,20}$, questionário econômico ${ }^{21}$, questionário de autoimagem e autoestima de Steglich $^{22}$,WHOQOL-BREF ${ }^{23}$ e WHOQOL-OLD ${ }^{24}$.

$\mathrm{O}$ questionário de autoimagem e autoestima de Steglich ${ }^{22}$ é composto por 78 perguntas, divididas em quatro aspectos fundamentais: orgânico, social, intelectual e emocional. Essas questões são separadas entre as que avaliam a autoestima e a autoimagem. Sendo assim, pelo escopo desta pesquisa, optou-se por utilizar somente as questões relacionadas à autoimagem.

O questionário WHOQOL-BREF possui 26 questões, divididas em quatro domínios: físico, psicológico, relações sociais e meio ambiente ${ }^{23}$. Já o WHOQOL-OLD é um instrumento específico para avaliar a qualidade de vida de idosos e é complementar ao WHOQOL-BREF. É composto por seis domínios: autonomia, funcionamento do sensório, atividades do passado, presente e futuro, participação social, intimidade e morte e morrer ${ }^{24}$.

\section{Análise de dados}

Foi realizado um cálculo amostral, a priori. Utilizou-se o software GPower 3.1.9.4, a fim de encontrar um tamanho do efeito $f(0,43)$, adotando-se os valores 0,05 para alfa e 0,80 para beta. $O$ número total da amostra foi estimado em 57 participantes, aproximadamente 20 pessoas por grupo. Assumindo possíveis desistências ou erros ao responder os questionários, optou-se por coletar 30 pessoas por grupo.

A descrição dos participantes foi realizada mediante medidas de tendência central e dispersão para variáveis contínuas e distribuição de frequência absoluta e relativa para variáveis categóricas. A normalidade e homogeneidade foram avaliadas com os testes Shapiro Wilk e Bartllet's test.

As análises comparativas foram executadas por meio de Anova para um fator. As comparações múltiplas foram realizadas por meio do post hoc de Bonferroni e a significância estatística de $\mathrm{p}<0,05$ foi adotada para todas as análises. As comparações para as variáveis categóricas foram realizadas com utilização do teste qui-quadrado. Todas as análises foram feitas utilizando o software estatístico Stata MP 14.1.

\section{RESULTADOS E DISCUSSÃO}

A idade média da amostra foi de 72,10 $\pm 7,93$ anos (mínimo: 60 anos; máximo: 91 anos). A faixa etária de 60 a 65 anos foi a com maior participação. O tempo de prática 
de pilates pelos participantes foi de aproximadamente 38 meses, e de hidroginástica, de 74 meses. Os dados sobre as características sociodemográficas dos voluntários estão dispostos na Tabela 1 .

A amostra foi composta em sua maioria por participantes do sexo feminino $(75,27 \%)$, informação que colabora com a feminização da velhice ${ }^{25,26}$. A cor/raça predominante foi a branca $(86,02 \%)$. Em relação ao estado civil, a maioria dos participantes é casada (64,52\%). Um estudo realizado em Campinas, São Paulo, mostrou que a maioria dos homens idosos da amostra era casada, porém a maior parte das mulheres eram viúvas ${ }^{27}$, o que corrobora na compreensão do maior número de mulheres da amostra.

A aposentadoria configurou a maior parte da amostra da pesquisa $(73,12 \%)$. A classe econômica em sua maioria foi a A $(34,41 \%)$. O grau de escolaridade com maior proporção foi o superior completo $(27,96 \%)$, o que pode estar associado ao elevado nível socioeconômico da amostra, já que isso contribui na busca e melhoria do processo de estudo formal.

Tabela 1. Características sociodemográficas dos participantes $(n=93)$

\begin{tabular}{|c|c|c|}
\hline Variáveis & $\mathrm{n}$ & $\%$ \\
\hline \multicolumn{3}{|l|}{ Sexo } \\
\hline Feminino & 70 & 75,27 \\
\hline Masculino & 23 & 24,73 \\
\hline \multicolumn{3}{|l|}{ Cor/raça } \\
\hline Branca & 80 & 86,02 \\
\hline Preta & 1 & 1,08 \\
\hline Amarela & 4 & 4,30 \\
\hline Parda & 8 & 8,60 \\
\hline \multicolumn{3}{|l|}{ Faixa etária } \\
\hline De 60 a 65 anos & 25 & 26,88 \\
\hline De 66 a 72 anos & 24 & 25,81 \\
\hline De 73 a 78 anos & 21 & 22,58 \\
\hline De 79 a 85 anos & 18 & 19,35 \\
\hline De 86 a 91 anos & 5 & 5,38 \\
\hline \multicolumn{3}{|l|}{ Estado civil } \\
\hline Solteiro (a) & 2 & 2,15 \\
\hline Casado (a) & 60 & 64,52 \\
\hline Separado (a)/ Divorciado (a) & 5 & 5,38 \\
\hline Viúvo (a) & 26 & 27,96 \\
\hline \multicolumn{3}{|l|}{ Escolaridade } \\
\hline Primário incompleto & 8 & 8,60 \\
\hline Primário completo / Ens. fund. incompleto & 10 & 10,75 \\
\hline Ens. fund. completo / Ens. méd. incompleto & 7 & 7,53 \\
\hline Ens. méd. completo / Curso sup. incompleto & 15 & 16,13 \\
\hline Curso superior completo & 26 & 27,96 \\
\hline Curso técnico completo & 4 & 4,30 \\
\hline Pós-graduação & 23 & 24,73 \\
\hline
\end{tabular}

Tabela 1. Continuação

\begin{tabular}{|c|c|c|c|c|}
\hline Variáveis & & & $\mathrm{n}$ & $\%$ \\
\hline \multicolumn{5}{|l|}{ Classificação econômica } \\
\hline A & & & 32 & 34,41 \\
\hline B1 & & & 16 & 17,20 \\
\hline B2 & & & 26 & 27,96 \\
\hline $\mathrm{Cl}$ & & & 8 & 8,60 \\
\hline C2 & & & 9 & 9,68 \\
\hline$D-E$ & & & 2 & 2,15 \\
\hline \multicolumn{5}{|l|}{ Ocupação atual } \\
\hline Aposentado & & & 68 & 73,12 \\
\hline Pensionista & & & 8 & 8,60 \\
\hline Dona de casa & & & 7 & 7,53 \\
\hline Outro & & & 10 & 10,75 \\
\hline Variáveis & Min & Máx & Média & DP \\
\hline $\begin{array}{l}\text { Tempo de prática de pilates } \\
\text { (em meses) }\end{array}$ & 1 & 144 & 38,13 & 41,53 \\
\hline $\begin{array}{l}\text { Tempo de prática de } \\
\text { hidroginástica (em meses) }\end{array}$ & 1 & 300 & 74,25 & 99,63 \\
\hline
\end{tabular}

Fonte: Elaboração própria.

n: número; Mín: mínimo; Máx: máximo; DP: desvio-padrão.

A Tabela 2, a seguir, apresenta as comparações das proporções da autoimagem (baixa ou elevada) entre os grupos. Nenhuma diferença significativa foi verificada $\left(\mathrm{X}^{2}=3,72, \mathrm{p}=0,15\right)$.

Tabela 2. Comparação e frequência da percepção de autoimagem entre os grupos de pilates, hidroginástica e não praticantes ( $n=93)$

\begin{tabular}{|c|c|c|c|c|c|}
\hline \multirow[b]{2}{*}{$\begin{array}{c}\text { Classes de } \\
\text { autoimagem }\end{array}$} & $\begin{array}{l}\text { Pilates } \\
(n=31)\end{array}$ & $\begin{array}{l}\text { Hidroginástica } \\
\qquad(\mathrm{n}=30)\end{array}$ & $\begin{array}{c}\text { Não } \\
\text { praticantes } \\
(n=32)\end{array}$ & \multirow[b]{2}{*}{$\mathrm{X}^{2}$} & \multirow[b]{2}{*}{ p } \\
\hline & n (\%) & n (\%) & $n(\%)$ & & \\
\hline Baixa & $20(64,52)$ & $20(66,67)$ & $27(84,38)$ & \multirow{2}{*}{3,72} & \multirow{2}{*}{0,15} \\
\hline Elevada & $11(35,48)$ & $10(33,33)$ & $5(15,62)$ & & \\
\hline \multicolumn{3}{|c|}{ Classes de autoimagem } & n & \multicolumn{2}{|c|}{$\%$} \\
\hline \multicolumn{3}{|c|}{$\begin{array}{l}\text { Baixa } \\
\text { Elevada }\end{array}$} & 67 & \multicolumn{2}{|c|}{$\begin{array}{l}72,04 \\
27,96\end{array}$} \\
\hline
\end{tabular}

Fonte: Elaboração própria.

$X^{2}$ : teste de qui-quadrado; $n$ : número.

Observa-se que o número de participantes com baixa autoimagem é alto, 67 no total (72,04\%), mas que entre os grupos, o que obteve maior número de pessoas com autoimagem baixa foi o de não praticantes. Isso pode ocorrer porque o esquema corporal é a relação de aspectos psicológicos, sociológicos e fisiológicos e a autoimagem é formada desde o nascimento por meio do controle motor. Dessa maneira, a autoimagem é ligada ao movimento e à ação ${ }^{8}$.

Apesar de o resultado não ter sido significativo nessa amostra, compreender a autoimagem dos idosos é importante, pois conhecer a relação da população idosa com o próprio corpo e suas implicações é crucial para 
melhor entender esse grupo e, assim, estimular a prática de um envelhecimento com mais qualidade ${ }^{28}$.

Por meio de uma revisão integrativa, percebeu-se que poucos instrumentos validados são usados para a compressão da autoimagem, pois exceto o questionário de Steglich só apareceram mais dois instrumentos: a escala de silhuetas de Stunkard e a escala de imagem corporal/ BIS (body imagem scale). Esse fato demonstra que são poucas as ferramentas propostas a avaliar a autoimagem, especialmente em idosos ${ }^{14}$.

A Tabela 3 apresenta os resultados das comparações da percepção da qualidade de vida avaliada pelo WHOQOLBREF entre os idosos dos grupos. A análise de variância identificou diferenças significativas entre os grupos para o domínio ambiental $\left(\mathrm{F}_{(2,90)}=3,32, \mathrm{p}=0,04\right)$, que foram refutadas após o post hoc de Bonferroni $(\mathrm{p}=0,08)$, que demonstrou somente uma tendência de diferença entre os grupos. É possível que esse valor no teste post hoc tenha ocorrido pelo limitado número da amostra.

Apesar disso, todas as médias dos escores, tanto da qualidade de vida geral, quanto dos domínios do WHOQOL-BREF, foram menores no grupo de não praticantes. Entretanto, é interessante observar que a maior média do grupo não praticante foi no domínio psicológico, quase se igualando aos demais grupos. O domínio psicológico compreende questões como sentimentos positivos e negativos, pensar, aprender, memória e concentração, autoestima, imagem corporal e aparência, espiritualidade, religião e crenças pessoais ${ }^{23}$. Como a amostra de não praticantes foi de uma comunidade religiosa, talvez o aspecto de espiritualidade, religião e crenças pessoais possa ter contribuído para a alta média desse domínio.

Tabela 3. Comparação da percepção de qualidade de vida avaliada pelo WHOQOL-BREF entre os grupos de pilates, hidroginástica e não praticantes

\begin{tabular}{lcccccc}
\multicolumn{7}{c}{ WHOQOL-BREF } \\
\cline { 1 - 5 } & $\begin{array}{c}\text { Pilates } \\
(\mathrm{n}=31)\end{array}$ & $\begin{array}{c}\text { Hidroginástica } \\
(\mathrm{n}=\mathbf{3 0})\end{array}$ & $\begin{array}{c}\text { Não } \\
\text { praticantes } \\
(\mathrm{n}=32)\end{array}$ & & \\
\cline { 2 - 5 } & Média $\pm \mathrm{DP}$ & Média $\pm \mathrm{DP}$ & Média $\pm \mathrm{DP}$ & $\mathrm{F}$ & $\mathbf{p}$ \\
QVG & $76,61 \pm 15,72$ & $75,83 \pm 15,37$ & $69,14 \pm 17,95$ & 1,97 & 0,14 \\
Físico & $71,54 \pm 15,19$ & $71,07 \pm 13,86$ & $63,50 \pm 16,09$ & 2,81 & 0,06 \\
Psicológico & $71,91 \pm 12,45$ & $72,50 \pm 11,40$ & $72,13 \pm 12,22$ & 0,02 & 0,98 \\
Social & $67,74 \pm 18,47$ & $62,22 \pm 20,73$ & $61,98 \pm 17,05$ & 0,94 & 0,39 \\
Ambiental & $74,70 \pm 13,60^{*}$ & $74,79 \pm 13,15^{*}$ & $67,38 \pm 12,50$ & 3,32 & 0,04
\end{tabular}

Fonte: Elaboração própria.

QVG: qualidade de vida geral; DP: desvio-padrão; *indica tendências de diferenças em comparação ao grupo de não praticantes $(p=0,08)$.

As comparações realizadas na percepção da qualidade de vida avaliada pelo WHOQOL-OLD entre os grupos estão dispostas na Tabela 4. Foi possível verificar diferenças significativas entre os grupos para os domínios de autonomia $\left(\mathrm{F}_{(2,90)}=7,46, \mathrm{p}=0,001\right)$ e morte e morrer $\left(\mathrm{F}_{(2,90)}=4,46, \mathrm{p}=0,01\right)$.

Tabela 4. Comparação da percepção da qualidade de vida avaliada pelo WHOQOL-OLD entre os grupos de pilates, hidroginástica e não praticantes $(\mathrm{n}=93)$

\begin{tabular}{|c|c|c|c|c|c|}
\hline \multicolumn{6}{|c|}{ WHOQOL-OLD } \\
\hline & $\begin{array}{l}\text { Pilates } \\
(n=31)\end{array}$ & $\begin{array}{l}\text { Hidroginástica } \\
\qquad(n=30)\end{array}$ & $\begin{array}{c}\text { Não } \\
\text { praticantes } \\
(n=32)\end{array}$ & & \\
\hline & Média \pm DP & Média \pm DP & Média $\pm \mathrm{DP}$ & $\mathbf{F}$ & $p$ \\
\hline QVG & $68,95 \pm 13,75$ & $68,26 \pm 12,19$ & $65,79 \pm 13,11$ & 0,51 & 0,60 \\
\hline FS & $81,25 \pm 15,14$ & $77,70 \pm 20,21$ & $70,50 \pm 21,37$ & 2,59 & 0,08 \\
\hline AUT & $70,56 \pm 16,39$ & $73,95 \pm 14,31^{b}$ & $58,59 \pm 18,49^{a b}$ & 7,46 & 0,001 \\
\hline PPF & $72,78 \pm 13,45$ & $72,29 \pm 12,78$ & $65,23 \pm 15,48$ & 2,88 & 0,06 \\
\hline PSO & $50,16 \pm 15,78$ & $50,33 \pm 12,17$ & $47,03 \pm 13,61$ & 0,56 & 0,57 \\
\hline MEM & $60,28 \pm 25,28^{a}$ & $63,54 \pm 24,40$ & $77,34 \pm 22,54^{a}$ & 4,46 & 0,01 \\
\hline INT & $63,10 \pm 33,40$ & $56,25 \pm 33,63$ & $61,91 \pm 33,69$ & 0,36 & 0,69 \\
\hline
\end{tabular}

Fonte: Elaboração própria.

QVG: qualidade de vida geral; FS: funcionamento do sensório; AUT: autonomia; PPF: passado, presente e futuro; PSO: participação social; MEM: morte e morrer; INT: intimidade; DP: desviopadrão; Caracteres iguais denotam diferença significativa entre os grupos $(p<0,05)$.

No domínio de autonomia, os grupos de pilates e hidroginástica apresentaram maiores valores de percepção de qualidade de vida em comparação ao grupo de não praticantes. $\mathrm{O}$ domínio autonomia reflete a liberdade para tomar decisões próprias, sentir-se no controle do futuro, sentir que as pessoas ao redor respeitam sua liberdade e ser capaz de fazer as atividades que gosta ${ }^{24}$. No estudo de Ferreti et al. $^{29}$, constatou-se que o grupo de praticantes de atividade física apresentou uma melhor percepção de qualidade de vida, quando comparado ao grupo de não praticantes, após avaliação por meio do WHOQOL-BREF e OLD.

A manutenção da condição física proporcionada pela prática de atividades corporais, principalmente por pessoas idosas, é um fator muito importante para a manutenção da vida e da autonomia do indivíduo ${ }^{29}$. Dessa forma, pode-se sugerir que os grupos pilates e hidroginástica apresentaram diferença significativa nesse domínio por estarem ativos fisicamente e dependerem menos de outras pessoas na manutenção de vida diária.

Já para o domínio de morte e morrer, os indivíduos do grupo não praticantes apresentaram maiores valores de percepção de qualidade de vida em comparação aos praticantes de pilates. O domínio morte e morrer consiste na preocupação sobre a maneira como a pessoa irá morrer, medo de não ser capaz de controlar a própria morte, medo de morrer e de sentir dor antes de morrer $^{24}$. O tema morte possui uma forte relação com as crenças pessoais, espiritualidade e religião, constructos que fazem parte do domínio psicológico do WHOQOL-BREF, e que também apresentou maior média do grupo de não praticantes. 
O fato de a coleta de dados do grupo de não praticantes ter sido feita em uma comunidade religiosa pode explicar o motivo da diferença significativa no domínio morte e morrer, quando comparado ao grupo pilates. A religião tem um papel importante para muitas pessoas, influenciando comportamentos e identidades, principalmente na fase idosa, quando a trajetória de vida começa a ser repensada e analisada, o que pode interferir na saúde física e mental do indivíduo ${ }^{30}$. Sugere-se então que ser participante de uma comunidade religiosa e dedicar-se às questões espirituais pode levar a pessoa a ter uma melhor percepção sobre a própria morte.

Este trabalho apresentou algumas limitações, como o número limitado da amostra, a falta de um espaço adequado para a coleta dos dados, a diversidade de estúdios de pilates coparticipantes, com diferentes metodologias e instrutores, o que pode alterar a percepção corporal dos alunos e a dificuldade em encontrar um instrumento de avaliação de autoimagem corporal compatível com o estudo.

\section{CONSIDERAÇÕES FINAIS}

A pesquisa revelou que a comparação da autoimagem entre os grupos não apresentou nenhuma diferença significativa, apesar de o número de participantes com baixa autoimagem ter sido alto.

Nos domínios da qualidade de vida, os grupos pilates e hidroginástica apresentaram maiores valores de média, em geral. No WHOQOL-OLD, verificaram-se diferenças significativas no domínio autonomia entre os grupos pilates e hidroginástica quando comparados ao grupo não praticantes. No domínio morte e morrer, os maiores valores foram no grupo não praticantes em relação ao grupo pilates.

Os resultados desta pesquisa sugerem que a prática de atividade física pode ter beneficiado essa população idosa, promovendo uma melhora na qualidade de vida, porém não isoladamente, visto que os valores médios dos escores não foram tão diferentes entre os grupos pilates e hidroginástica em relação ao grupo não praticante, assim como somente alguns domínios apresentaram resultado estatístico significativo.

\section{REFERÊNCIAS}

1. Tavares RE, Jesus MCP, Machado DR, Braga VAS, Tocantins FR, Merighi MAB. Envelhecimento saudável na perspectiva de idosos: uma revisão integrativa. Rev Bras Geriatr Gerontol. 2017;20(6):889-900. doi: 10.1590/1981-22562017020.170091.

2. Instituto Brasileiro de Geografia e Estatística. Projeção da população 2018: número de habitantes do país deve parar de crescer em 2047 [Internet]. 2018 [cited 2018 Nov 12]. Available from : https://bit.ly/3A4eLai

3. Veras RP, Oliveira M. Envelhecer no Brasil: a construção de um modelo de cuidado. Cienc Saude Colet. 2018; 23(6):1929-36. doi: 10.1590/1413-81232018236.04722018.

4. Barros DD. Imagem corporal: a descoberta de si mesmo. Hist Cienc Saude. 2005;12(2):547-54. doi: 10.1590/ S0104-59702005000200020.

5. Teixeira INDO, Guariento ME. Biologia do envelhecimento: teorias, mecanismos e perspectivas. Cien Saude Colet. 2010;15(6):2845-57. doi: 10.1590/S1413-81232010000600022.

6. Mosquera JJM, Stobäus CD. Auto-imagem, auto-estima e auto-realização: qualidade de vida na universidade. Psicol Saude Doenças [Internet]. 2006;7(1):83-8 [cited 2006 Jul 30]. Available from: https://bit.ly/3fmEoLD

7. Feldenkrais M. Consciência pelo movimento: exercícios fáceis de fazer para melhorar a postura, visão, imaginação e percepção de si mesmo. São Paulo: Summus; 1977.

8. Scatolin HG. A imagem do corpo: as energias construtivas da psique. Psicol Rev [Internet]. 2012;21(1):115-20 [cited 2021 Aug 2]. Available from: https://bit.ly/3fiSFsH

9. Pereira EF, Teixeira CS, Santos A. Qualidade de vida: abordagens, conceitos e avaliação. Rev Bras Educ Fis Esp. 2012;26(2):241-50. doi: 10.1590/S1807-55092012000200007.

10. Paschoal SMP. Qualidade de vida na velhice. In: Freitas EV, Py L, editors. Tratado de geriatria e gerontologia. 2 nd ed. Rio de Janeiro: Guanabara Koogan; 2006. p. 148-53.

11. Almeida MAB, Gutierrez GL, Marques R. Qualidade de vida: definição, conceitos e interfaces com outras áreas de pesquisa. São Paulo: EACH/USP; 2012.

12. Pilates JH. A obra completa de Joseph Pilates: sua saúde e o retorno à vida pela contrologia. São Paulo: Phorte; 2010.

13. Pilates JH, Miller WJ. Fundamentos básicos de uma educação física natural. In: Pilates JH. A obra completa de Joseph Pilates: sua saúde e o retorno à vida pela contrologia. São Paulo: Phorte; 2010. p. 117-37.

14. Copatti SL, Kuczmainski AG, Tombini FF, Sá CA. Imagem corporal e autoestima em idosos: uma revisão integrativa da literatura. Estud Interdiscip Envelhec [Internet]. 2017;22(3):4762 [cited 2021 Aug 2]. Available from: https://bit.ly/3lhsUgq

15. Bolsanello DP. A educação somática e os conceitos de descondicionamento gestual, autenticidade somática e tecnologia interna. Motrivivência. 2011;(36):306-22. doi: 10.5007/2175-8042.2011v23n36p306.

16. Caldwell K, Adams M, Quin R, Harrison M, Greeson J. Pilates, mindfulness and somatic education. J Dance Somat Pract [Internet]. 2013;5(2):141-53 [cited 2021 Aug 2]. Available from: https://bit.ly/3A27fgs

17. Sampieri RH, Collado CF, Lucio MPB, editors. Metodologia de pesquisa. 5th ed. Porto Alegre: Penso; 2013. 
18. Gil AC. Métodos e técnicas de pesquisa social. 6th ed. São Paulo: Atlas; 2008.

19. Veras R, Dutra S. Perfil do idoso brasileiro: questionário BOAS. Rio de Janeiro: Uerj/Unati; 2008.

20. Mazo GZ. Atividade física e qualidade de vida de mulheres idosas [dissertation]. Porto: Universidade do Porto; 2003.

21. Associação Brasileira de Empresas de Pesquisa. Critério de classificação econômica do Brasil. São Paulo: Associação Brasileira de Empresas de Pesquisa; 2016.

22. Steglich LA. Terceira idade, aposentadoria, auto-imagem e auto-estima [master's thesis]. Porto Alegre: Universidade Federal do Rio Grande do Sul; 1978.

23. Fleck MPA, Louzada S, Xavier M, Chachamovich E, Vieira $G$, Santos L, et al. Aplicação da versão em português do instrumento abreviado de avaliação da qualidade de vida "WHOQOL-BREF". Rev Saude Publica. 2000;34(2):178-83. doi: 10.1590/S0034-89102000000200012.

24. Fleck MP, Chachamovich E, Trentini C. Desenvolvimento e validação da versão em português do módulo WHOQOLOLD. Rev Saude Publica. 2006;40(5):785-91. doi: 10.1590/ S0034-89102006000600007.
25. Almeida AV, Mafra SCT, Silva EP, Kanso S. A feminização da velhice: em foco as características socioeconômicas, pessoais e familiares das idosas e o risco social. Textos Contextos. 2015;14(1):115-131. doi: 10.15448/1677-9509.2015.1.19830.

26. Xirocostas ZA, Everingham SE, Moles AT. The sex with the reduced sex chromosome dies earlier: a comparison across the tree of life. Biol Lett. 2019;16(3):1-6. doi: 10.1098/rsbl.2019.0867.

27. Sousa NFS, Lima MG, Cesar CLG, Barros MBA. Envelhecimento ativo: prevalência e diferenças de gênero e idade em estudo de base populacional. Cad Saude Publica. 2018;34(11):1-14. doi: 10.1590/0102-311x00173317.

28. Chaim J, Izzo H, Sera C. Cuidar em saúde: satisfação com imagem corporal e autoestima de idosos. Mundo Saude [Internet]. 2009;33(2):175-81 [cited 2021 Aug 2]. Available from: https://bit.ly/2WNwW5Z

29. FerretiF, Beskow GCT, Slaviero RC, Ribeiro CG. Análise da qualidade de vida em idosos praticantes e não praticantes de exercício físico regular. Estud Interdiscip Envelhec. 2015;20(3):729-43. doi: https://doi.org/10.22456/2316-2171.41384.

30. Oliveira RMF. A influência comportamental religiosa na qualidade de vida do método Pilates em idosos. In: Totum [Internet]. 2019;6(2):21 [cited 2021 Aug 2]. Available from: https://bit.ly/3zZO6eX 